\title{
MUC1 Antigen-Specific CD8 T Lymphocytes Targeting MCF7 and MDA-MB-231 Human Breast Adenocarcinoma Cell Lines
}

\author{
Panagiotis Parsonidis, Dimitrios-Athanasios Ntanovasilis, Ioannis Papasotiriou* \\ Research Genetic Cancer Centre SA (R.G.C.C. SA), Florina, Greece \\ Email: *office@rgcc-genlab.com, * papasotiriou.ioannis@rgcc-genlab.com.parsonidis.panagiotis@rgcc-genlab.com, \\ dimitrios.danovasilis@rgcc-genlab.com
}

How to cite this paper: Parsonidis, P., Ntanovasilis, D.-A. and Papasotiriou, I. (2019) MUC1 Antigen-Specific CD8 T Lymphocytes Targeting MCF7 and MDA-MB-231 Human Breast Adenocarcinoma Cell Lines. Journal of Cancer Therapy, 10, 495-509.

https://doi.org/10.4236/jct.2019.107041

Received: June 3, 2019

Accepted: July 6, 2019

Published: July 9, 2019

Copyright $\odot 2019$ by author(s) and Scientific Research Publishing Inc. This work is licensed under the Creative Commons Attribution International License (CC BY 4.0).

http://creativecommons.org/licenses/by/4.0/

\begin{abstract}
MUC1 is an antigen that is overexpressed on the cell surface of many human breast adenocarcinomas and other types of cancer. The cancer immunity cycle has seven steps, starting with release of cancer cell antigen and following with cancer antigen presentation. Priming, activation and trafficking of $\mathrm{T}$ cells to tumors are the next steps and the infiltration of $\mathrm{T}$ cells into tumors, the recognition of cancer cells by $\mathrm{T}$ cells and killing of cancer cells are the final steps. We have tested a synthetic peptide for the MUC1 antigen and generated dendritic cells (DCs) that were pulsed with the specific peptide. Mature DCs were used to activate naive $\mathrm{T}$ cells to differentiate into antigen-specific CTLs. CTLs were tested for proliferation, cytokine release (IFN $\gamma$ ), activation markers and cytotoxicity against human breast adenocarcinoma cell lines. The cytotoxic effect of those CTLs was higher against MCF7 human cell line than against MDA-MB-231 human cell line.
\end{abstract}

\section{Keywords}

MUC1, Dendritic Cells, Cytotoxic T Lymphocytes, Immunotherapy

\section{Introduction}

Breast cancer is the second leading cause of cancer death in women. Conventional treatment approaches, such as chemotherapy, radiotherapy, endocrine therapy and surgery, have become efficient over the last decades, but they still have limitations regarding the response and the development of resistance. The new approaches are moving towards the development of personalized, targeted therapies, including immunotherapies, which will minimize resistance with lower toxicity and higher specificity [1]. Therapy that is using the body's immune 
system has opened up new avenues for reducing death rates. Breast cancer was not originally thought to be immunogenic, but immunotherapy has had encouraging results in the past few years. Cancer immunotherapy is triggering patient's immune system to attack and kill tumor cells. Moreover, treatment can be used as an important addition to conventional therapies [2]. Adoptive immunotherapy is the ex vivo activation and expansion of tumor-specific immune cells and infusion of those cells into cancer patients. Cytotoxic T lymphocytes generated from naïve CD8 $\mathrm{T}$ cells seem to have potent cytotoxic activity and can be used in adoptive immunotherapy [3] [4]. Molecular identification of human cancer antigens and in vitro techniques, which are allowing generation of large numbers of dendritic cells (DCs) that are presenting cancer antigens to T cells, have given new prospects at the concept of cancer immunotherapy [5].

Immature DCs have high phagocytic activity and they are able to capture antigens. Delivery of antigens to DCs can be employed by several systems including viral vectors, defined peptides and undefined peptides, RNA from tumor cells, whole tumor lysates and fusion with tumor cells. Immature DCs can capture killed tumor cells and present their antigens. Antigen-presenting mature DCs are able to activate antigen-specific cytotoxic T lymphocytes [6]. Cytotoxic $\mathrm{T}$ lymphocyte (CTL), also known as $\mathrm{CD}^{+} \mathrm{T}$ cell, is a type of $\mathrm{T}$ lymphocyte that is important for immune defence against potentially threatening pathogens and cancer cells [7]. Tumor fitness is the prediction whether a cancer cell will be attacked by immune cells (T cells). Strong immune response against the tumor means that there is low tumor fitness [8]. High tumor fitness is related with loss of function of immune cells and an increase in the amount of checkpoint inhibitors such as cytotoxic T lymphocyte antigen 4 (CTLA4), programmed cell death protein 1 (PD1) and lymphocyte activation gene 3 protein (LAG3) that are markers of T cell exhaustion [9] [10] [11].

It is critical to identify the most potent stage of human DCs for designing successful DC-based immunotherapy protocol. Immature DCs are mainly located in peripheral tissues where they capture antigens. The stimuli is necessary to switch DCs to immunostimulatory mode. The maturation process is associated with changes in phenotype and function, with upregulation of adhesion molecules and expression of chemokine receptors [12]. Mature DCs are potent in priming naive CD8 $\mathrm{T}$ cells and in expanding memory CD8 T cells. Mature DCs can be used as immunotherapy adjuvants for priming and efficient differentiation of naive $\mathrm{CD} 8 \mathrm{~T}$ cells into cytotoxic $\mathrm{T}$ cells [13].

The epithelial mucin MUC1 is a highly glycosylated type I transmembrane glycoprotein that is overexpressed on the cell surface of many human breast and ovarian adenocarcinomas as well as on some multiple myelomas and B cell lymphomas, but not on the surface of normal cells. Its expression is associated with predominantly, but not exclusively, epithelial tumor cells. Abnormal expression of MUC1 has been associated with metastatic process of tumor cells and the expression is higher in progressive and metastatic tumor cells than in primary tumor cells. It is recognized as an attractive target for immunotherapeu- 
tic strategies for the past 30 years but clinical benefit for general application has not yet been achieved [14] [15]. MUC1 continues to arouse interest as a therapeutic target and there is an increase in MUC1-based clinical trials initiated in 2017 [16]. MUC1 $1_{79-87}$ TLAPATEPA is a non-variable number tandem repeat region MUC1-derived HLA-A* 02:01-restricted epitope that elicits peptide specific cytotoxic T lymphocyte (CTL) immunity. It can be used as component of peptide-based vaccines and can be used in research of anti-MUC1 CTL responses of patients with MUC1 positive tumors [17].

The present study aimed to extend the possibility of using MUC1-derived T cell epitopes to induce immunotherapeutic approaches. To prove the presentation of $\mathrm{T}$ cell epitopes by the cancer cells, induction of MUC1-specific CTLs was performed in vitro using peptide-pulsed DCs as antigen-presenting cells. Hence, evaluation of their functional status in terms of proliferation, cytotoxicity and cytokine release against two human breast adenocarcinoma cell lines was done.

\section{Materials and Methods}

\subsection{Cell Culture}

Cancer cell lines were purchased from ECACC (European Collection of Authenticated Cell Cultures) (Salisbury, UK). MCF7 (ECACC 86012803) human Caucasian breast adenocarcinoma cell line, that was luminal type (estrogen receptor positive, progesterone receptor positive, HER2 negative), was maintained in RPMI 1640 supplemented with $2 \mathrm{mM} \mathrm{L-glutamine,} \mathrm{10 \%} \mathrm{Foetal} \mathrm{Bovine} \mathrm{Serum}$ (FBS) and $1 \%$ MEM Non-essential amino acids (NEAA) in $37^{\circ} \mathrm{C} 5 \% \mathrm{CO}_{2}$ incubator. MDA-MB-231 (ECACC 92020424) human Caucasian breast adenocarcinoma, that was triple negative (estrogen receptor negative, progesterone receptor negative, HER2 negative), was maintained in RPMI 1640 supplemented with $2 \mathrm{mM}$ L-glutamine and $15 \%$ Foetal Bovine Serum (FBS) in $37^{\circ} \mathrm{C} 5 \% \mathrm{CO}_{2}$ incubator. RPMI 1640 Catalog\#R0883 was obtained from Sigma-Aldrich, Darmstadt, Germany, L-glutamine Catalog\#G7513-100ML was purchased from Sigma-Aldrich, Darmstadt, Germany and FBS Catalog\#FB-1001/500 was obtained from BioSera, Nuaille, France. MEM Non-essential amino acids Catalog\#M7145-100ML was purchased from Sigma-Aldrich, Darmstadt, Germany.

\subsection{Synthetic Peptide}

HLA-A* 02:01-binding peptide MUC1 $_{79-87}$ TLAPATEPA was used for in vitro stimulation of $\mathrm{T}$ cells and purchased from GenScript (Leiden, Netherlands). It was $>90 \%$ pure as indicated by analytical high-performance liquid chromatography.

\subsection{DCs Generation from Monocytes}

In order to generate DCs, monocytes were isolated by peripheral blood mononuclear cells of healthy donors (HD) (females, 25 - 30 years old) with Ficoll-seperation. Adherent monocytes were cultured with granulocyte-monocyte 
colony-stimulating factor Catalog\#11343123 and IL-4 Catalog\#11340043 (ImmunoTools, Friesoythe, Germany) in RPMI 1640 Medium Catalog\#R0883 (Sigma-Aldrich, Darmstadt, Germany) with 10\% heat-inactivated FBS and 1\% L-glutamine for 6 days. At day 6 of culture, DCs were pulsed with $\mathrm{MUC1}_{79-87}$ TLAPATEPA peptide at final concentration of $10 \mu \mathrm{g} / \mathrm{ml}$ and the addition of $\beta 2$ microglobulin Catalog\#C-69302 (PromoCell, Heidelberg, Germany) at final concentration of $3 \mu \mathrm{g} / \mathrm{ml}$ and incubation for $5 \mathrm{~h}$. Maturation of DC was induced with cytokines cocktail including IL-6 $(10 \mathrm{ng} / \mathrm{ml})$ Catalog\#11340060, IL-1 $\beta$ (25 $\mathrm{ng} / \mathrm{ml}$ ) Catalog\#11340012, TNF- $\alpha$ (50 ng/ml) Catalog\#11343013 (ImmunoTools, Friesoythe, Germany) and PGE2 (10 ${ }^{-6}$ M) Catalog\#2296/10 (R\&D Systems, Abingdon, $\mathrm{UK}$ ) and incubation for $48 \mathrm{~h}$ at $37^{\circ} \mathrm{C}$ and $5 \% \mathrm{CO}_{2}$. Freezing of two aliquots of mature DCs was performed for second and third stimulation of $\mathrm{T}$ cells [18].

\subsection{Generation of Antigen-Specific CTL}

Peripheral blood mononuclear cells (PBMCs) of healthy donors (HD) (females, 25 - 30 years old) were isolated with Ficoll-seperation (Biocoll separating solution-Catalog\#1077, Biochrom, Berlin, Germany). Monocytes could be separated from lymphocytes given their ability to adhere to the surface of the flask. Non-adherent T cells were collected and added in co-culture with mature DCs in a 12-well plate in OpTmizer ${ }^{\mathrm{TM}}$ CTS $^{\mathrm{TM}}$ T-Cell Expansion medium Catalog\#A1048501 (ThermoFischer Scientific, Darmstadt, Germany) with the addition of IL-2 (10 ng/ml) Catalog\#11340023 (ImmunoTools, Friesoythe, Germany). The ration of T cells to DCs was 30:1. At day 7 of co-culture, thawing of an aliquot of mature DCs for stimulation of T cells was done with the addition of IL-7 (5 ng/ml) Catalog\#11340072 (ImmunoTools, Friesoythe, Germany) and IL-2 (10 ng/ml). At day 14 of co-culture, thawing of the last aliquot of mature DCs and stimulation of $\mathrm{T}$ cells in the same way of first and second stimulation was done with the addition of IL-7 $(5 \mathrm{ng} / \mathrm{ml})$ and IL-2 $(10 \mathrm{ng} / \mathrm{ml})$. On day 21 of co-culture, CD8+ T cells were isolated from co-culture by positive selection with magnetic beads Catalog\#39-CD8-250 (Gentaur, Kampenhout, Belgium) and the cells were ready to be assessed for CTL activity [18].

\subsection{Immunophenotyping}

Flow cytometric analysis of monocyte derived DCs was performed by direct immunofluorescence of cell surface antigens using antibodies against CD11c conjugated with APC Catalog\#21487116 (ImmunoTools, Friesoythe, Germany), CD80 conjugated with PE Catalog\#21270804 (ImmunoTools, Friesoythe, Germany), CD83 conjugated with FITC Catalog\#MHCD8301 (ThermoFischer Scientific, Darmstadt, Germany) and CD86 conjugated with FITC Catalog\#21480863 (ImmunoTools, Friesoythe, Germany). Primed T cells were analyzed using antibodies against CD4 conjugated with PE Catalog\#21278044 (ImmunoTools, Friesoythe, Germany), CD8 conjugated with APC Catalog\#21620086 (ImmunoTools, Friesoythe, Germany), CD11a conjugated with APC Catalog\#21810116 (ImmunoTools, Friesoythe, Germany), CD69 conjugated with PE Cata- 
log\#12-0699-42 (ThermoFischer Scientific, Darmstadt, Germany) and CD25 Catalog\#555431 (BD Biosciences, San Jose, CA, USA) and CD62L Catalog\#ab222 (Abcam, Cambridge, UK) conjugated with FITC. Samples were analyzed on FACScan Calibur (Becton Dickinson, USA) and FSC 6 Express software.

\subsection{T Cell Proliferation}

$\mathrm{T}$ cell proliferation was assessed with CellTrace ${ }^{\mathrm{TM}}$ CFSE Cell Proliferation Kit Catalog\#C34554 (ThermoFischer Scientific, Darmstadt, Germany) according to the manufacturer's suggestions. After five days of co-culture with DCs, live T cells were analysed for Carboxyfluorescein Diacetate Succinimidyl Ester (CFSE) dilution by flow cytometry.

\subsection{Cytokine Production}

On days 6 and 12 of co-culture with mature DCs, supernatants were harvested and frozen at $-80^{\circ} \mathrm{C}$ until used. Levels of cytokine IFN- $\gamma$ were determined using an ELISA kit Catalog\#31673539 (ImmunoTools, Friesoythe, Germany) according to manufacturer's suggestions. Absorbance at $450 \mathrm{~nm}$ with reference wavelength at $605 \mathrm{~nm}$ was taken and the optical density was compared with the optical density of unstimulated $\mathrm{T}$ cells.

\subsection{LDH Cytotoxicity Detection Assay}

500 cancer cells were seeded in a U-bottom 96-well plate Catalog $\$ 4430200$ (Orange Scientific, Braine-I'Alleud, Belgium) and were left overnight at $37^{\circ} \mathrm{C}$ to adhere. Next day, pre-activated $\mathrm{T}$ cells were added at ratio 1:10 and co-culture for $20 \mathrm{hr}$ in final volume of $100 \mu \mathrm{l}$. Plates were centrifuged at $250 \mathrm{~g}$ for $10 \mathrm{mi}$ nutes and $50 \mu \mathrm{l}$ of supernatants were transferred into corresponding wells of an optically clear 96-well flat bottom microplate Catalog\#781722 (Brand, Wertheim, Germany). Level of cytotoxicity was measured with Cytotoxicity Detection Kit (LDH) Catalog\#11644793001 (Roche, Darmstadt, Germany). $50 \mu \mathrm{l}$ of substrate were added to the corresponding wells and microplate was left to incubate for 30 minutes at room temperature in the dark. Absorbance was measured at $490 \mathrm{~nm}$ using an ELISA reader and reference wavelength was at $605 \mathrm{~nm}$.

\subsection{Statistical Analysis}

One sample t-test was used to determine differences in the mean. $\mathrm{P}$ values $<0.05$ were considered to indicate a statistically significant difference.

\subsection{Ethics Approval}

All procedures were conducted according to the standards of Safety, Bioethics and Validation. The study was reviewed and approved by Bioethical Committee of the Research Genetic Cancer Centre Group. Each healthy donor (HD) provided informed consent in writing for the use of their sample in the present study. The healthy donors (HD) retained the right to withdraw their sample until the date when the sample was received at the laboratory and tested. 


\section{Results}

\subsection{Generation}

The generation of immature DCs and the progress of the development of mature DCs was reviewed by phase-contrast inverted microscopy (Figure 1). At the beginning of the culture, monocytes were spherical and adherent to the surface of the flask. At day 5, immature DCs were found forming clusters suspending in culture medium. Upon maturation, DCs were transformed into large irregular cells with cytoplasmic projections.

\subsection{Cell Surface Activation Markers Indicating Mature Phenotype of DCs}

Flow cytometric analysis of DCs revealed significant differences in the expression of surface molecules (Figure 2). DCs were gated according to forward (FSC) and side (SSC) scatter profile. Immature DCs showed a substantially enhanced expression of CD11c, low expression of CD80, CD86 and very low expression of CD83. On the other hand, mature DCs showed high expression of CD86 and CD83 and were positive for CD80 (Table 1).

\subsection{Stimulation of T Cells by MUC1-Pulsed DCs}

The ability of mature DCs to stimulate $\mathrm{T}$ cells proliferation was determined by

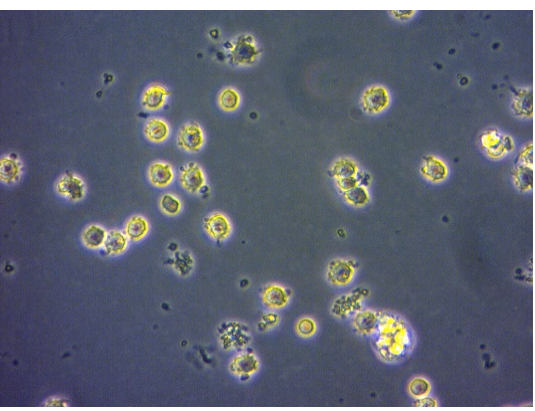

(A)

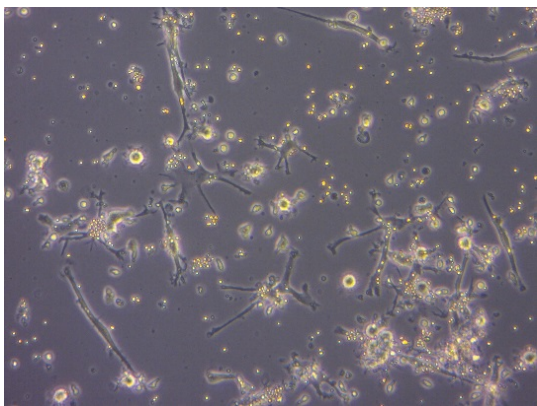

(C)

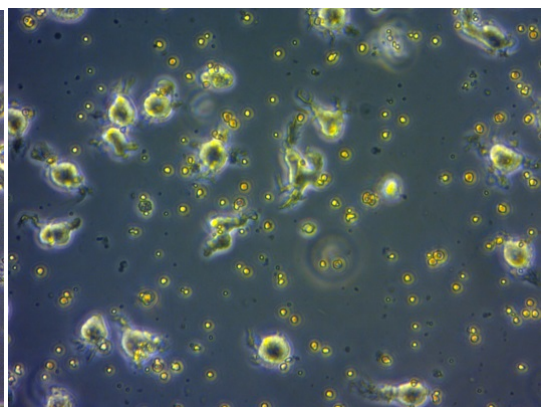

(B)

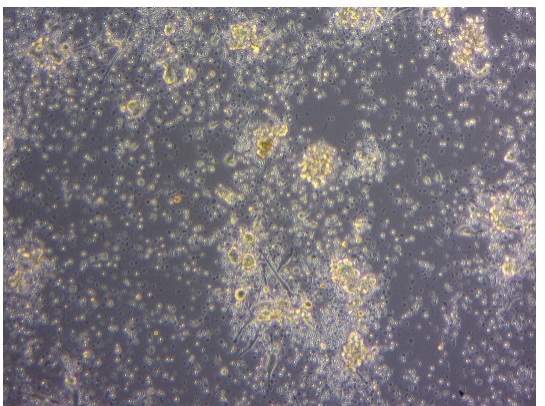

(D)

Figure 1. Dendritic cell generation. Microscope images (A) Day 0, Monocytes for DC generation (magnification, $\times 20$ ), (B) Day 6, immature DCs (GM-CSF, IL-4) (magnification, $\times 40)$ and (C) Day 8 , mature DCs (TNF $\alpha$, IL-1 $\beta$, IL-6, PGE2) (magnification, $\times 10$ ). Progress of the development of DCs. (D) Day 12, Mature DCs co-culture with T cells (magnification, $\times 10$ ). 

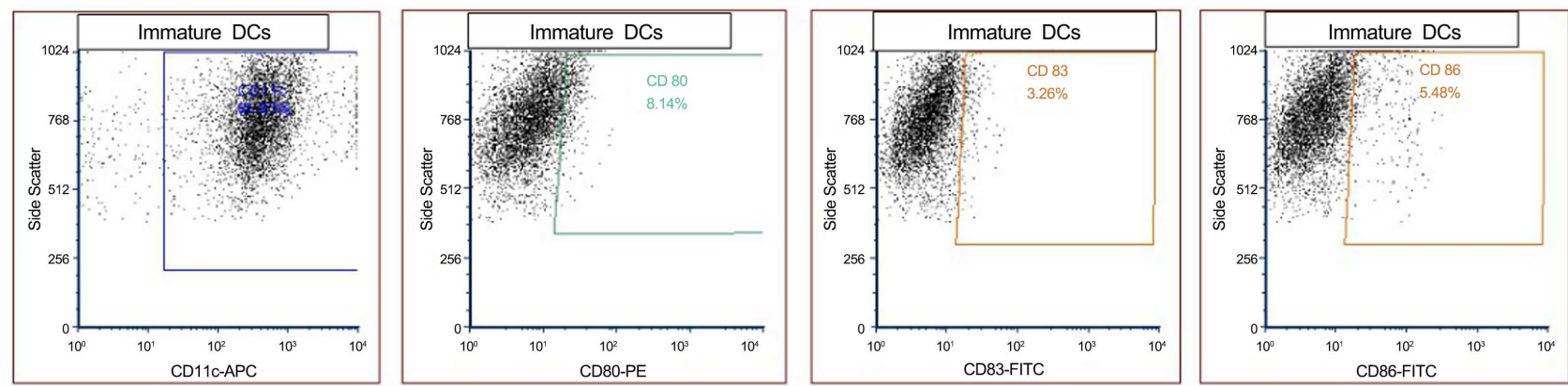

(A)
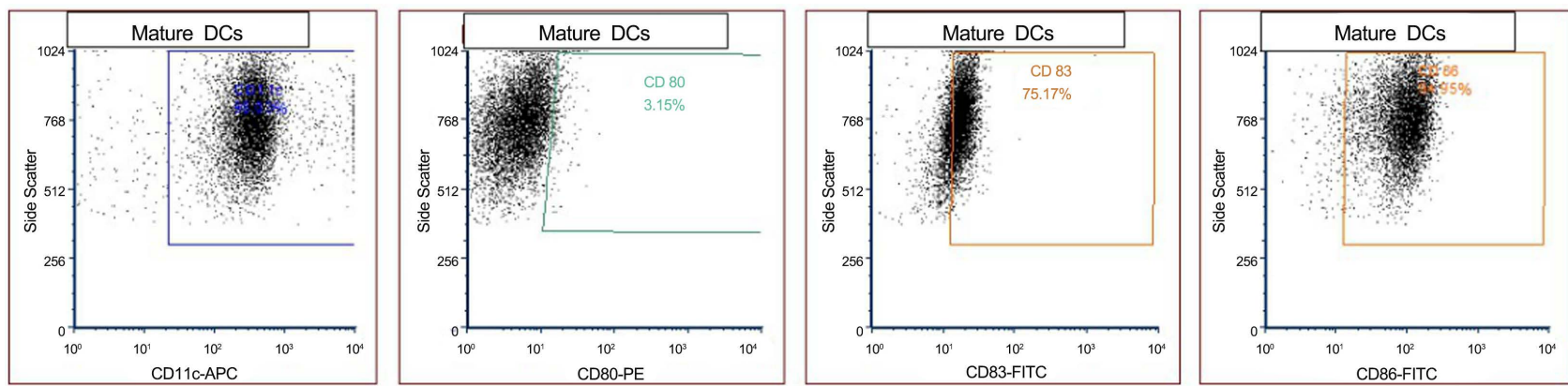

(B)

Figure 2. Gating was performed on dot plots by plotting the Side Scatter parameter against the channel of each corresponding fluorochrome. Row (A) shows the populations of immature dendritic cells while row (B) shows the mature dendritic cells.

Table 1. Percentages of expression of cell surface activation markers indicating the stage of maturation of DCs. Immature DCs and DCs that have been treated with maturation cocktail. CD11c-APC, CD80-PE, CD83-FITC, CD86-FITC.

\begin{tabular}{cllll}
\hline & \multicolumn{5}{c}{ DCs Phenotype } \\
\hline & CD11c & CD80 & CD83 & CD86 \\
\hline Immature DCs & $97.33 \%$ & $7.56 \%$ & $3.26 \%$ & $5.48 \%$ \\
DCs (Maturation cocktail) & $93.29 \%$ & $3.15 \%$ & $75.17 \%$ & $94.95 \%$ \\
\hline
\end{tabular}

CFSE assay. Every generation of cells is presented with a different subset on flow cytometry (Figure 3). As controls, unstimulated T cells cultured alone, showed the CFSE intensity of non-divided cells, while non-labeled cells showed the auto-fluorescence of the cells and the limits of detectable cell divisions. 1 CFSE peak could be seen in the panel of unstimulated CD8 $\mathrm{T}$ cells, indicating that the cells have not undergone up to any division, while 4 CFSE peaks could be seen in the panel of stimulated CD8 T cells, indicating that cells have been divided up to three generations. Similarly, only one CFSE peak could be seen in the panel of unstimulated CD4 T cells, showing no division, while three CFSE peaks were detected in the panel of stimulated CD4 T cells, indicating that the cells have been divided up to two generations.

\subsection{Cytokine IFN- $\gamma$ Release by Stimulated T Cells}

$\mathrm{T}$ cells were in culture alone, in co-culture with MUC1-pulsed DCs and in 

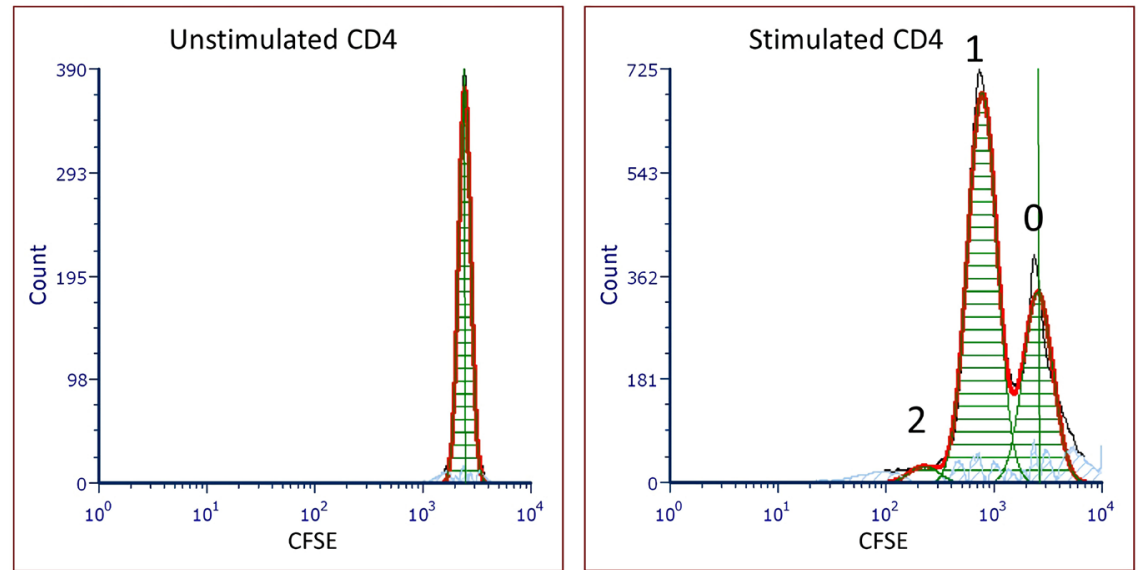

(A)
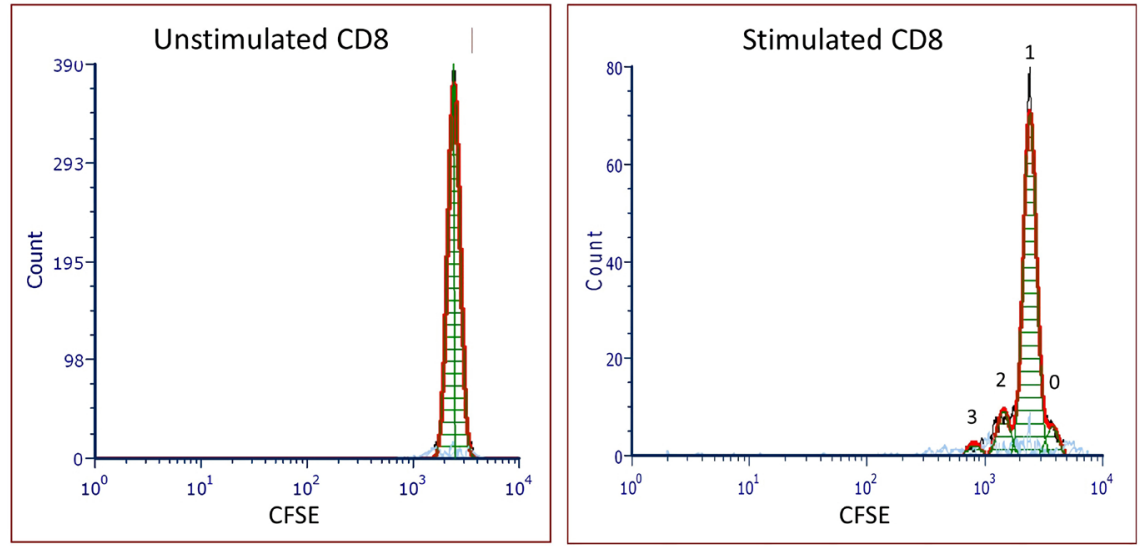

(B)

Figure 3. Data was analysed using the proliferation plot on FCS Express. The software was able to recognize the first generation (undivided cells) and count the divided cells' generations. Row (A) shows the CD4 cells and Row (B) the CD8 cells.

co-culture with DCs that had not been pulsed with any peptide. Supernatants from all the above cultures harvested and analyzed with ELISA for IFN $\gamma$ release. When comparing the release of IFN $\gamma$ between $\mathrm{T}$ cells alone and $\mathrm{T}$ cells that had been in co-cultivation with MUC1-pulsed DCs, there was a statistically significant higher expression of IFN $\gamma$ in both 6 and 12 days, in the supernatants of MUC1 primed T cells. A statistically significant difference was also observed between the culture of T cells with MUC1-pulsed DCs and T cells cultures with DCs that had not been pulsed in the samples harvested at 12 days. IFN $\gamma$ release was higher in the co-cultivation with MUC1-pulsed DCs (Figure 4).

\subsection{Cell Surface Markers Displaying Activation of Cytotoxic T Lymphocytes}

The activation of cytotoxic $\mathrm{T}$ lymphocytes was assessed by flow cytometry against four markers (Figure 5). The expression of CD11a was much higher on stimulated cells than on unstimulated $\mathrm{T}$ cells, reaching $72.04 \%$, while on unstimulated the expression was $38.46 \%$. For the rest of the markers, there was a 


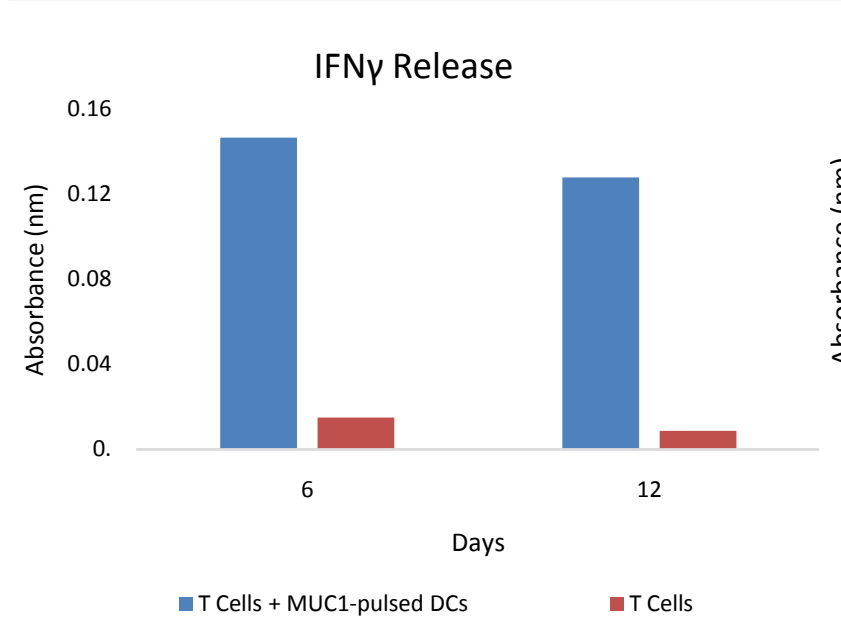

(A)
IFNy Release

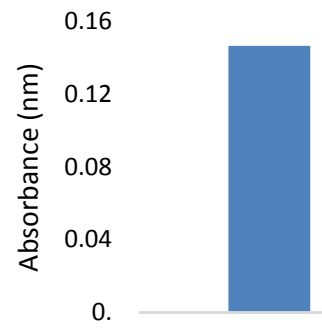

6

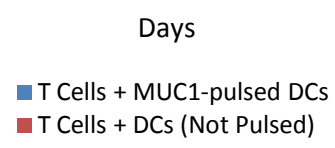

(B)

Figure 4. IFN $\gamma$ release by $\mathrm{T}$ cells that have been stimulated by MUC1-pulsed DCs compared with (A) T cells that had no stimulation and (B) T cells that had been co-cultivated with DCs that had not been pulsed with any antigen.
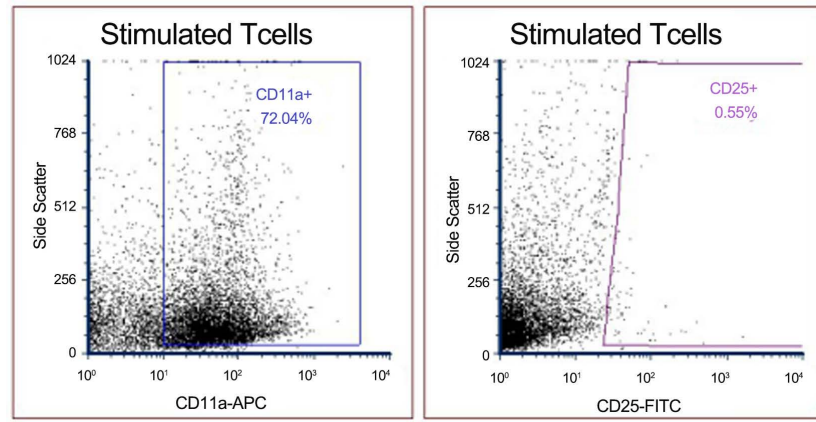

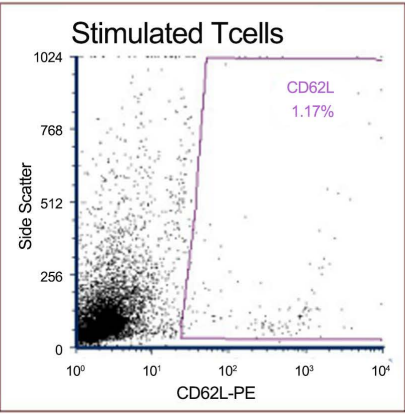

(A)
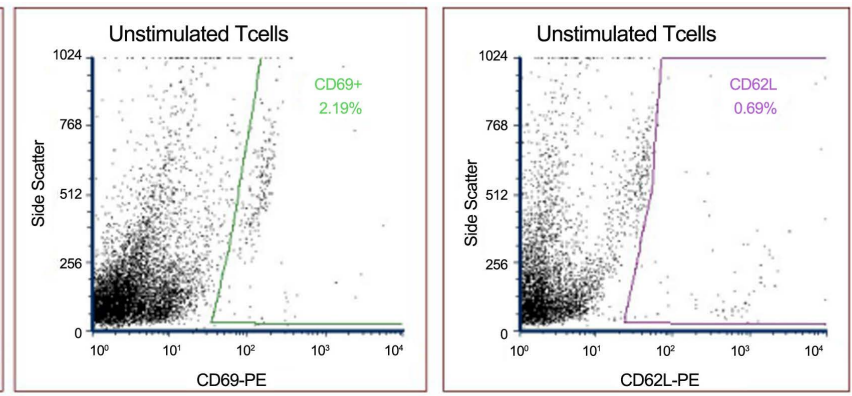

(B)

Figure 5. Gating strategy for calculating the percentages for the expression of cytotoxic T lymphocyte activation markers. Row (A) shows stimulated $\mathrm{T}$ cells and Row (B) unstimulated T cells.

slightly higher value in the expression on the stimulated $\mathrm{T}$ cells than on the cells that had not been stimulated. Stimulated $\mathrm{T}$ cells had a very low expression of CD25-0.55\%, CD69-2.25\% and CD62L-1.17\%, while the unstimulated $\mathrm{T}$ cells exhibited even lower expression of CD25-0.29\%, CD69-2.19\% and CD62L-0.69\%.

\subsection{Detection of MUC1-Specific T Cell Cytotoxic Activity}

We assessed the cytotoxic activity of antigen-specific CTLs with LDH Cytotoxic- 
ity detection assay. Released LDH in supernatant was measured with a $30 \mathrm{~min}$ coupled enzymatic assay that resulted in the conversion of a tetrazolium salt into a red formazan product.

The percentage of cytotoxicity has been calculated as (experimental valuespontaneous effector cell release-spontaneous target cell release)/(maximum target cell release-spontaneous target cell release) $\times 100 \%$. Background value had been subtracted from all the above values. MCF7 and MDA-MB-231 (human breast adenocarcinoma cell lines) were served as target cells. The percentage of cytotoxicity was $44.9 \%$ against MCF7 and 33.3\% against MDA-MB-231 at an E:T (effector:target) ratio of 10:1 (Figure 6).

\section{Discussion}

The present study was designed to identify cytotoxic T lymphocyte generation from MUC1 antigen-pulsed dendritic cells and the cytotoxic activity of those CTLs against MCF7 and MDA-MB-231 human breast adenocarcinoma cell lines. This therapeutic modality could be considered as a vaccination strategy after in vivo testing to fully humanized mice models to determine the efficacy and toxicity. The efficiency of tumor antigen processing and presentation by human DCs depends on the source or form of tumor-derived materials, the maturation of DCs and the responsiveness of $\mathrm{T}$ cell population for stimulation with DCs [19].

Adoptive immunotherapy depends on activation and expansion of cytotoxic $\mathrm{T}$ lymphocytes that are going to fight against the tumor. Isolation and purification of immune cell population is very crucial for the development of efficient immunotherapy. Naïve CD8+ T cells display low levels of inhibitory immune checkpoint receptors and have resistance patterns against $\mathrm{T}$ cell exhaustion. Hence, CTLs with naïve CD8+ T cell origin have strong potency to indicate high

57.50

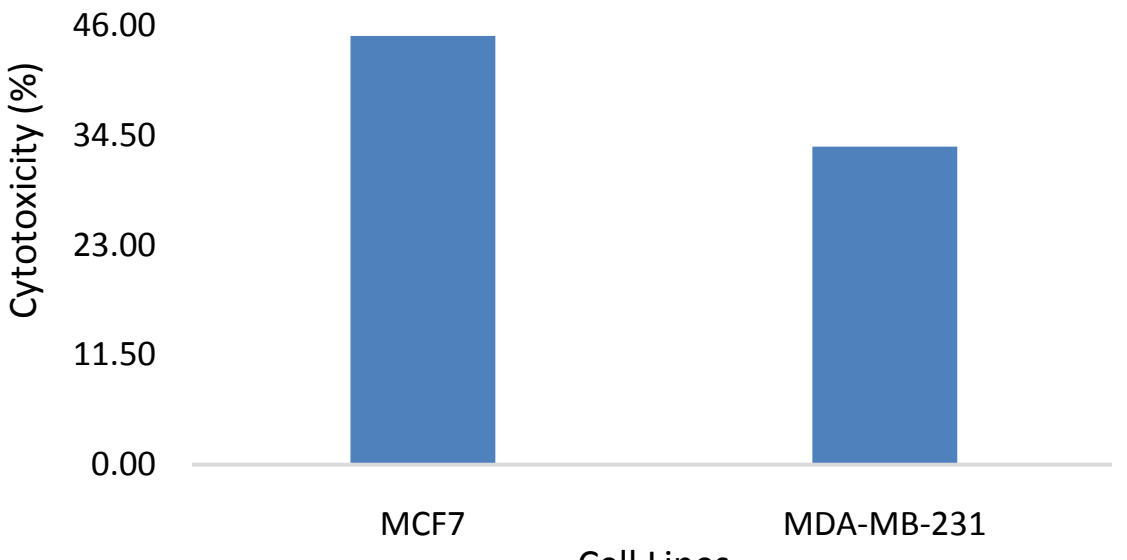

Cell Lines

Figure 6. Percentage of cytotoxicity results of MUC1-specific CTLs against MCF7 and MDA-MB-231 by LDH Cytotoxicity Detection Assay. 
cytotoxic activity against tumor cells [3]. One promising area in cancer immunotherapy is T cell co-culture with antigen-pulsed DCs. One mature DC is able to efficiently activate approximately $100 \mathrm{~T}$ cells, whereupon they can trigger an immune response. In vitro co-culturing of $\mathrm{T}$ cells and DCs could be used to avoid the negative influence of tumor growth products that are depressing the functional activity of mature antigen-pulsed DCs administered to the patient [20].

Mature DCs are significantly better at CTL induction due to higher expression of MHC and costimulatory molecules than CTL induction by immature DCs. The presentation of tumor antigens by immature DCs may lead to tolerance induction in the absence of proper co-stimulation. In peripheral lymphoid organs immature DCs are not able to elicit CTL responses. Mature DCs express high levels of co-stimulatory molecules, resulting in priming of antigen-specific CTLs, if there is sufficient co-administration with antigens to stimulate DCs activation [21] [22].

Mature DCs were able to induce the full complement of $\mathrm{T}$ cell activation, IFN $\gamma$ production, $\mathrm{T}$ cell proliferation and development of cytotoxic $\mathrm{T}$ cells [23]. Naïve CD8 T cells do not produce large amounts of IFN- $\gamma$, but differentiation in CTLs is triggering the production of higher levels of the cytokine [24] [25]. In the present study, we observed that activated T cells with MUC1-pulsed DCs produced higher levels of IFN $\gamma$ cytokine than naive CD8 $\mathrm{T}$ cells. This was the first sign that we could have achieved successful differentiation of naive CD8 T cells in CTLs. Moreover, IFN $\gamma$ production was higher in T cells that were stimulated with MUC1-pulsed DCs than those that were stimulated with mature DCs that had not been pulsed with any peptide. It might be a result suggesting the specificity of differentiated CTLs against MUC1 antigen.

Loaded DCs with tumor antigens can induce CD8 $\mathrm{T}$ cell proliferation. It was observed that DCs loaded with MUC1 peptide induced CD8 T cell proliferation and naive CD8 $\mathrm{T}$ cells that were used as control had not undergone up to any divisions. Co-cultivation of CD8 T cells with mature DCs and the addition of IL-2 is necessary to induce maximal proliferation of purified CD8 T cells [26]. Naive CD4 $\mathrm{T}$ cells had no signs of proliferation and activated CD4 $\mathrm{T}$ cells showed lower levels of proliferation than activated CD8 T cells. CD8 $\mathrm{T}$ cells become responsive to low doses of IL-2 quicker than CD4 T cells [27].

CD11a plays a critical role in $\mathrm{T}$ cell infiltration and activation. Change in the frequency of CD11a expression can be used to track antigen-specific CD4 and CD8 $\mathrm{T}$ cell responses followed $\mathrm{T}$ cell-targeted vaccination. It is crucial for the optimal expansion of antigen-specific CD4 and CD8 T cells [28]. CD25 plays a role in the proliferation and survival of activated $\mathrm{T}$ lymphocytes. It is expressed at low levels in resting lymphocytes and it is increased upon activation in a time-dependent manner, remaining elevated until 24 hours and declining thereafter [29]. In the present study, the analysis has been performed many days after the activation signal and it was clear that CD25 expression was at very low levels. CD69 is another early marker of lymphocyte activation due to its rapid 
appearance on the surface of the plasma after stimulation [30]. Similarly to CD25, the expression of CD69 by the stimulated T cells with MUC1-pulsed DCs was very low. CD62L is shed from the cell membrane following $\mathrm{T}$ cell activation. Naive CD8 T cells are showing higher expression of CD62L, but the expression is decreased when $\mathrm{T}$ cell differentiate into effector phase [31]. Higher CD62L expression is correlated with central memory $\mathrm{T}$ cell phenotype. The slight increase that we observed in the expression of CD62L by stimulated $\mathrm{T}$ cells might be the result of the extent of cell division that might be associated with the extent of memory formation.

After stimulation of cytotoxic T cells with antigen-pulsed DCs, LDH cytotoxicity test was performed to study the cytotoxic activity of MUC1 antigen-specific CTLs against human breast adenocarcinoma cell lines. Chromium $\left({ }^{51} \mathrm{Cr}\right)$ release assay has been widely used for detecting the cytolytic activity of CTLs. Despite the sensitivity and efficiency of the assay, this method has a great drawback, the use of radioactive materials, which is related with environmental safety concerns and inconvenience to handle [32]. LDH cytotoxicity detection assay is a non-radioactive alternative to chromium release assay and is based on detection of cytosolic enzyme that is released in culture medium from the cytosol of damaged cells. It is a colorimetric assay for quantification of cell lysis and death [33]. LDH results demonstrated that MUC1-specific CTLs had the ability to induce cytotoxicity over human breast adenocarcinoma cell lines. MCF7 and MDA-MB-231 were two cell lines that had different characteristics, MCF7 was luminal type breast cancer and MDA-MB-231 was triple negative breast cancer, resulting in different resistance to CTLs. CTLs that had been activated against $\mathrm{MUC1}_{79-87}$ TLAPATEPA peptide, demonstrated greater cytotoxic effect in MCF7 cell line than in MDA-MB-231 cell line.

Further studies are needed to determine the potential therapeutic value of cytotoxic $\mathrm{T}$ lymphocytes that are generated by antigen-pulsed DCs. In vitro results have to be compared with in vivo results for safety, efficacy and validation that there are no side effects following that specific immunotherapy is crucial. Identification of shared cancer antigens that potentially might be presented by DCs and could generate CTLs with high cytotoxicity activity, is very important for the standardization of the protocols and the development of a valid therapeutic proposal.

\section{Conclusion}

Pulsing of DCs with antigenic epitopes and the use of those DCs in activation of naïve CD8 T cells to become cytotoxic T lymphocytes may be considered a novel approach to adoptive immunotherapy.

\section{Acknowledgements}

This research did not receive any specific grant. The authors declare that there is no conflict of interest regarding the publication of this article. 


\section{Conflicts of Interest}

The authors declare no conflicts of interest regarding the publication of this paper.

\section{References}

[1] Seliger, B. (2018) Immunotherapy of Breast Cancer. Breast Care, 13, 5-6. https://doi.org/10.1159/000486927

[2] Farkona, S., Diamandis, E.P. and Blasutig, I.M. (2016) Cancer Immunotherapy: The Beginning of the End of Cancer? BMC Medicine, 14, 73.

https://doi.org/10.1186/s12916-016-0623-5

[3] Nguyen, H.H., Kim, T., Song, S.Y., Park, S., Cho, H.H., Jung, S.H., Ahn, J.S., Kim, H.J., Lee, J.J., Kim, H.O., Cho, J.H. and Yang, D.H. (2016) Naïve CD8 ${ }^{+}$T Cell Derived Tumor-Specific Cytotoxic Effectors as a Potential Remedy for Overcoming TGF- $\beta$ Immunosuppression in the Tumor Microenvironment. Scientific Reports, 6 , Article No. 28208. https://doi.org/10.1038/srep28208

[4] Perret, R. and Ronchese, F. (2008) Effector CD8 ${ }^{+}$T Cells Activated in Vitro Confer Immediate and Long-Term Tumor Protection in Vivo. European Journal of Immunology, 38, 2886-2895. https://doi.org/10.1002/eji.200838483

[5] Neidhardt-Berard, E.M., Berard, F., Banchereau, J. and Palucka, A.K. (2004) Dendritic Cells Loaded with Killed Breast Cancer Cells Induce Differentiation of Tumor-Specific Cytotoxic T Lymphocytes. Breast Cancer Research, 6, R322-R328. https://doi.org/10.1186/bcr794

[6] Nouri-Shirazi, M., Banchereau, J., Bell, D., Burkeholder, S., Kraus, E.T., Davoust, J. and Palucka, K.A. (2000) Dendritic Cells Capture Killed Tumor Cells and Present Their Antigens to Elicit Tumor-Specific Immune Responses. The Journal of Immunology, 165, 3797-3803. https://doi.org/10.4049/jimmunol.165.7.3797

[7] Andersen, M.H., Schrama, D., Straten, P. and Becker, J.C. (2006) Cytotoxic T Cells. Journal of Investigative Dermatology, 126, 32-41. https://doi.org/10.1038/sj.jid.5700001

[8] Sarkizova, S. and Hacohen, N. (2017) How T Cells Spot Tumour Cells. Nature, 551, 444-446. https://doi.org/10.1038/d41586-017-07267-9

[9] Baitsch, L., Legat, A., Barba, L., Fuertes Marraco, S.A., Rivals, J.P., Baumgaertner, P., Christiansen-Jucht, C., Bouzourene, H., Rimoldi, D., Pircher, H., Rufer, N., Matter, M., Michielin, O. and Speiser, D.E. (2012) Extended Co-Expression of Inhibitory Receptors by Human CD8 T-Cells Depending on Differentiation, Antigen-Specificity and Anatomical Localization. PLOS ONE, 7, e30852. https://doi.org/10.1371/journal.pone.0030852

[10] Wherry, E.J. (2011) T Cell Exhaustion. Nature Immunology, 12, 492-499. https://doi.org/10.1038/ni.2035

[11] Crespo, J., Sun, H., Welling, T.H., Tian, Z. and Zou, W. (2013) T Cell Anergy, Exhaustion, Senescence, and Stemness in the Tumor Microenvironment. Current Opinion in Immunology, 25, 214-221. https://doi.org/10.1016/j.coi.2012.12.003

[12] Hoffmann, T., Meidenbauer, N., Dworacki, G., Kanaya, H. and Whiteside, T.L. (2000) Generation of Tumor-Specific T Lymphocytes by Cross-Priming with $\mathrm{Hu}$ man Dendritic Cells Ingesting Apoptotic Tumor Cells. Immunology, 60, 13.

[13] Pufnock, J.S., Cigal, M., Rolczynski, L.S., Andersen-Nissen, E., Wolfl, M., McElrath, M.J. and Greenberg, P.D. (2011) Priming $\mathrm{CD}^{+} \mathrm{T}$ Cells with Dendritic Cells Ma- 
tured Using TLR4 and TLR7/8 Ligands Together Enhances Generation of CD8 ${ }^{+} \mathrm{T}$ Cells Retaining CD28. Blood, 117, 6542-6551.

https://doi.org/10.1182/blood-2010-11-317966

[14] Deicher, A., Andersson, R., Tingstedt, B., Lindell, G., Bauden, M. and Ansar, D. (2018) Targeting Dendritic Cells in Pancreatic Ductal Adenocarcinoma. Cancer Cell International, 18, 85. https://doi.org/10.1186/s12935-018-0585-0

[15] Gong, J., Apostolopoulos, V., Chen, D., Chen, H., Koido, S., Gendler, S.J., Mckenzie, I.F. and Kufe, D. (2000) Selection and Characterization of MUC1-SPECIFIC CD8 ${ }^{+}$ T Cells from MUC1 Transgenic Mice Immunized with Dendritic-Carcinoma Fusion Cells. Immunology, 101, 316-324. https://doi.org/10.1046/j.1365-2567.2000.00101.x

[16] Taylor-Papadimitriou, J., Burchell, J.M., Graham, R. and Beatson, R. (2018) Latest Developments in MUC1 Immunotherapy. Biochemical Society Transactions, 46, 659-668. https://doi.org/10.1042/BST20170400

[17] Heukamp, L.C., Van Der Burg, S.H., Drijfhout, J.-W., Melief, C.J.M., Taylor-Papadimitriou, J. and Offringa, R. (2001) Identification of Three Non-VNTR MUC1-Derived HLA-A* 0201-Restricted T-Cell Epitopes that Induce Protective Anti-Tumor Immunity in HLA/A2/ $\mathrm{K}^{\mathrm{b}}$-Transgenic Mice. International Journal of Cancer, 91, 385-392. https://doi.org/10.1002/1097-0215(200002)9999:9999<::AID-IJC1051>3.0.CO;2-Z

[18] Ranieri, E. (2014) Cytotoxic T-Cells: Methods and Protocols. In: Methods in Molecular Biology, Springer, Berlin, Heidelberg. https://doi.org/10.1007/978-1-4939-1158-5

[19] Fu, C. and Jiang, A. (2018) Dendritic Cells and CD8 T Cell Immunity in Tumor Microenvironment. Frontiers in Immunology, 9, 3059.

https://doi.org/10.3389/fimmu.2018.03059

[20] Obleukhova, I., Kiryishina, N., Falaleeva, S., Opatnikova, J., Kurilin, V., Kozlov, V., Vitsin, A., Cherkasov, A., Kulikova, E. and Sennikov, S. (2018) Use of Antigen-Primed Dendritic Cells for Inducing Antitumor Immune Responses in Vitro in Patients with Non-Small Cell Lung Cancer. Oncology Letters, 15, 1297-1306. https://doi.org/10.3892/ol.2017.7403

[21] Koido, S., Homma, S., Hara, E., Namiki, Y., Ohkusa, T., Gong, J. and Tajiri, H. (2010) Antigen-Specific Polyclonal Cytotoxic T Lymphocytes Induced by Fusion of Dendritic Cells and Tumor Cells. Journal of Biomedicine and Biotechnology, 2010, Article ID: 752381. https://doi.org/10.1155/2010/752381

[22] Kroger, C.J., Amoah, S. and Alexander-Miller, M.A. (2008) Cutting Edge: Dendritic Cells Prime a High Avidity CTL Response Independent of the Level of Presented Antigen. The Journal of Immunology, 180, 5784-5788. https://doi.org/10.4049/jimmunol.180.9.5784

[23] Kaiser, A., Bercovici, N., Abastado, J.P. and Nardin, A. (2003) Naive CD8 ${ }^{+}$T Cell Recruitment and Proliferation Are Dependent on Stage of Dendritic Cell Maturation. European Journal of Immunology, 33, 162-171.

https://doi.org/10.1002/immu.200390019

[24] Truxova, I., Kasikova, L., Hensler, M., Skapa, P., Laco, J., Pecen, L., Belicova, L., Praznovec, I., Halaska, M.J., Brtnicky, T., Salkova, E., Rob, L., Kodet, R., Goc, J., Sautes-Fridman, C., Fridman, W.H., Ryska, A., Galluzzi, L., Spisek, R. and Fucikova, J. (2018) Mature Dendritic Cells Correlate with Favorable Immune Infiltrate and Improved Prognosis in Ovarian Carcinoma Patients. Journal for ImmunoTherapy of Cancer, 6, 139. https://doi.org/10.1186/s40425-018-0446-3 
[25] De Araujo-Souza, P.S., Hanschke, S.C.H. and Viola, J.P.B. (2015) Epigenetic Control of Interferon-Gamma Expression in CD8 T Cells. Journal of Immunology Research, 2015, Article ID: 849573. https://doi.org/10.1155/2015/849573

[26] Geginat, J., Lanzavecchia, A. and Sallusto, F. (2003) Proliferation and Differentiation Potential of Human $\mathrm{CD}^{+}$Memory T-Cell Subsets in Response to Antigen or Homeostatic Cytokines. Blood, 101, 4260-4266.

https://doi.org/10.1182/blood-2002-11-3577

[27] Au-Yeung, B.B., Smith, G.A., Mueller, J.L., Heyn, C.S., Jaszczak, R.G., Weiss, A. and Zikherman, J. (2017) IL-2 Modulates the TCR Signaling Threshold for CD8 but Not CD4 T Cell Proliferation on a Single-Cell Level. The Journal of Immunology, 198, 2445-2456. https://doi.org/10.4049/jimmunol.1601453

[28] Christiaansen, A.F., Dixit, U.G., Coler, R.N., Beckmann, A.M., Reed, S.G., Winokur, P.L., Zimmerman, M.B., Varga, S.M. and Wilson, M.E. (2017) CD11a and CD49d Enhance the Detection of Antigen-Specific T Cells Following Human Vaccination. Vaccine, 35, 4255-4261. https://doi.org/10.1016/j.vaccine.2017.06.013

[29] Bajnok, A., Ivanova, M., Rigo Jr., J. and Toldi, G. (2017) The Distribution of Activation Markers and Selections on Peripheral T Lymphocytes in Preeclampsia. Mediators of Inflammation, 2017, Article ID: 8045161. https://doi.org/10.1155/2017/8045161

[30] Walsh, D.A., Hamilton, S.E. and Jameson, S.C. (2016) The Role of CD69 in Generating Resident Memory CD8 ${ }^{+} \mathrm{T}$ Cells. The Journal of Immunology, 196, 133.8.

[31] Schlub, T.E., Badovinac, V.P., Sabel, J.T., Harty, J.T. and Davenport, M.P. (2009) Predicting CD62L Expression during the $\mathrm{CD}^{+} \mathrm{T}$ Cell Response in Vivo. Immunology \& Cell Biology, 88, 157-164. https://doi.org/10.1038/icb.2009.80

[32] Fu, X., Tao, L., Rivera, A., Williamson, S., Song, X.T., Ahmed, N. and Zhang, X. (2010) A Simple and Sensitive Method for Measuring Tumor-Specific T Cell Cytotoxicity. PLOS ONE, 5, e11867. https://doi.org/10.1371/journal.pone.0011867

[33] Zaritskaya, L., Shurin, M.R., Sayers, T.J. and Malyguine, A.M. (2010) New Flow Cytometric Assays for Monitoring Cell-Mediated Cytotoxicity. Expert Review of Vaccines, 9, 601-616. https://doi.org/10.1586/erv.10.49 